\title{
Postoperative Delirium after Urological Surgery: A Literature Review
}

\author{
Ioannis Leotsakos ${ }^{a, b} \quad$ Ioannis Katafigiotis ${ }^{a, b} \quad$ Ofer N. Gofrit ${ }^{b} \quad$ Mordechai Duvdevani $^{b}$ \\ Dionysios Mitropoulos ${ }^{\mathrm{a}}$
}

a1st Department of Urology, University of Athens Medical School, Athens, Greece; 'bepartment of Urology, Hadassah Hebrew University Medical Center, Jerusalem, Israel

\section{Key Words}

Postoperative delirium • Predictor • Urological surgery • Endourology

\begin{abstract}
Purpose: We aimed to thoroughly search and identify studies referring to risk factors associated with postoperative delirium (POD) in patients undergoing open as well as endoscopic urological surgery. Methods: The review after a systematic literature search included 5 studies. Results: The incidence of POD was reported to be between 7.8 and $30 \%$ depending on the type of the urologic surgery, while in the majority of the studies the onset happened on the first postoperative day and the symptoms lasted $3 \pm 0.8$ days. Seventeen different risk factors for POD were identified and presented in detail. Conclusion: The Mini-Mental State Examination score and older age were significantly associated with the development of POD. However, the Confusion Assessment Method is very well validated against the diagnosis of delirium from the specialists. @ 2019 The Author(s)

Published by S. Karger AG, Basel
\end{abstract}

\section{Introduction}

Delirium is an acute confusional state characterized by a disturbance of consciousness, reduced ability to maintain attention and memory impairment, and known to occur usually in the elderly people [1]. The motoric presentation of delirium may vary from an apathetic, inactive state to an agitated, hyperactive state.

Postoperative delirium (POD) has a deep impact on the healthcare of patients and thus on the healthcare financial management. It is associated with increased lengths of hospital stay, costs, morbidity, and mortality $[2,3]$. However, until now few studies of POD included patients undergoing urologic surgery.

Prevention of POD remains challenging due to numerous potential risk factors. Also, it is challenging to apply all predictive factors to clinical practice without a welltrained patient care team. Therefore, an easy and simple method to evaluate the possibility of POD is required. POD incidences are reported to be $8.8 \%$ in general urological surgery [4], 29\% in radical cystectomy [5], and $21 \%$ in transurethral resection of prostate (TURP) [6].

Ioannis Leotsako

Endourology and Laparoscopic Urology

Hadassah Ein-Kerem University Hospital

The Hebrew University

Hadassah, P.O.B 12000

IL-91120 Jerusalem (Israel)

E-Mail j_leot@yahoo.gr 


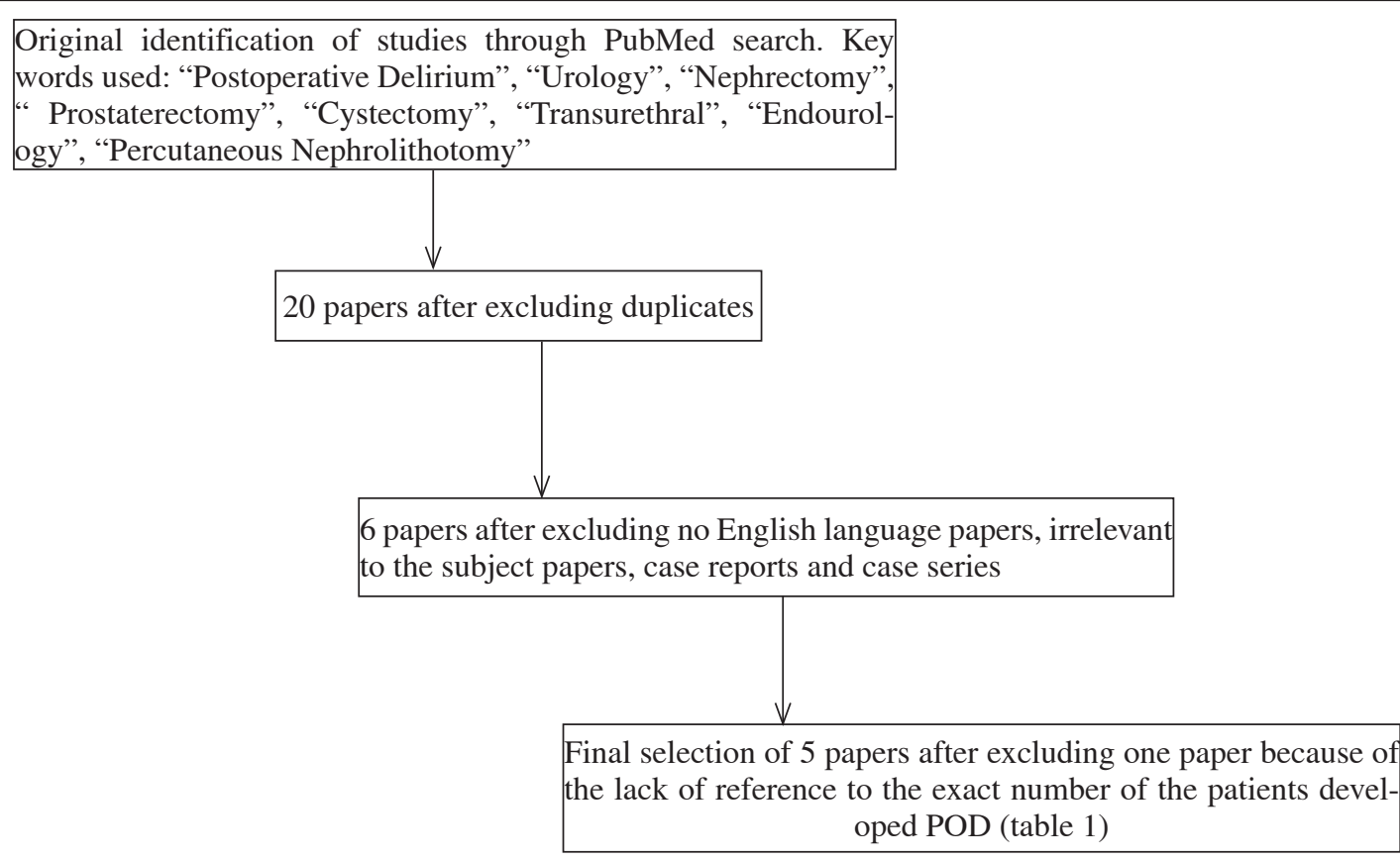

Fig. 1. Flowchart of the literature search.

We performed a literature review to identify studies referring to pre-, intra- and post-operative predictors for developing POD in patients undergoing open as well as endoscopic urological surgery.

\section{Method and Research Strategy}

A thorough PubMed-based research was conducted using the key word "postoperative delirium" in combination successively with the terms "urology", "transurethral", "nephrectomy", "prostatectomy", "cystectomy", "endourology", and "percutaneous nephrolithotomy" (fig. 1). Inclusion criteria were papers referring to POD in urologic operations in the English language. Exclusion criteria were case reports, case series and studies which included delirium in their evaluation regarding postoperative complications without POD and its potential risk factors being the main subject of the study. The initial combination search of the terms "postoperative delirium" and "urology" yielded 20 papers, while no additional papers occurred from the rest of the terms. The papers were evaluated independently by 2 authors (I.L. and I.K.) who concluded to 6 relevant to the subject papers. One paper was excluded because even though the writers evaluated the postoperative cognitive function measuring a total delirium score of the patients, there was no mention of the number of patients that probably developed a delirium. Finally, 5 papers [4-8] were considered eligible. All references are derived from the included studies in our review.

\section{Evaluation of Preoperative Cognitive Status and POD}

The preoperative cognitive status was evaluated by Sato et al. [7] with the frailty assessment. Frailty was measured by handgrip strength, fatigue scale of depression, fall risk assessment, and gait speed (the timed Get-up and Go test). Thus, they statistically established an optimal cut-off value for the timed Get-up and Go test at 13.0 seconds [7].

Patients with severe functional or cognitive impairment such as Alzheimer's disease (unable to answer functional or cognitive questions), vision disorder or hearing loss, pre-existing apparent dementia and cognitive loss, poor general health, medical conditions likely to result in death within a few months, or any other reasons for which patients were unable to perform physical tests or answer the questionnaire regarding fatigue scale were excluded from the study of Sato et al. [7]. Also, obvious demented people were excluded as well, mostly because these patients were not indicated for urological surgery.

In the other 4 studies [4-6,8], preoperative cognitive status was mostly evaluated using the Mini-Mental State Examination (MMSE). Tai et al. [6] administered the psychological questionnaire, including the MMSE [9], the Yesavage's Geriatric Depression Scale (GDS) short version, the Mendez calculation score 
Table 1. Presentation of the studies

\begin{tabular}{llcccc}
\hline Studies & Type of study & Patients, $\mathrm{n}$ & POD, $\%$ & Occurrence (postoperative day) & Duration, days \\
\hline Tognoni et al. [4] & prospective & 90 & 8.8 & 1 & $3.0 \pm 0.8$ \\
Large et al. [5] & prospective & 49 & 29 & day $1: \mathrm{n}=8$, days $5-7: \mathrm{n}=6$ & $1-5$ \\
Tai et al. [6] & prospective & 485 & 21.23 & 1 & $1-3$ \\
Sato et al. [7]. & prospective & 215 & 4.7 & day $1: \mathrm{n}=21$, day $2: \mathrm{n}=7$ \\
Xue et al. [8] & prospective & 358 & 7.8 & & - \\
\hline
\end{tabular}

Clock Drawing Test (CDT), and functional assessment, as the Activities of Daily Living (ADL) and Instrumental Activities of Daily Living (IADL).

The patients were evaluated postoperatively for delirium using the Confusion Assessment Method (CAM), according to the Diagnostic and Statistical Manual of Mental Disorders-V TR in most of the chosen studies [4-6,8]. CAM scores are determined by a diagnostic algorithm consisting of 4 clinical criteria: 1 ) acute onset or fluctuating course, 2) inattention, 3) disorganized or incoherent thinking, and 4) alteration in alertness. Diagnosis of delirium was made if the first and second criteria are present and either criterion 3 or 4 are present.

Delirium in one study [7] was diagnosed based on Diagnostic and Statistical Manual of Mental Disorders-V criteria [7, 10]: 1) There is a disturbance in attention and awareness (asking the same questions over and over and/or not be able to have a conversation); 2) Delirium develops over a short period, typically hours to days; 3) There is also another disturbance in cognition, such as in memory, orientation, language, and perception; 4) The disturbances in (1) and (3) are not better explained by another pre-existing, established, or evolving neurocognitive disorder (essential to the diagnosis of delirium is that the patient can respond to "verbal stimulation"); 5) There must also be evidence that the delirium is due to a direct physiological consequence of another medical condition, substance intoxication or withdrawal, or exposure to a toxin, or is due to multiple etiologies. If patients met any of these criteria, they were then diagnosed as a delirium.

\section{Presentation of the Studies}

In the study of Tognoni et al. [4], 56 patients who underwent open surgery and 34 endoscopic procedures were included. General anesthesia was employed in 68 patients while 22 had spinal anesthesia. Large et al. [5] studied 49 patients who underwent radical cystectomy with standard pelvic lymph node dissection performed with the patients under general endotracheal anesthesia without epidural analgesia. Tai et al. [6] studied 485 patients who underwent TURP under spinal (regional) anesthesia with the use of saline fluid for the prevention of TUR-syndrome (hyponatremia) and subsequently of POD. Delirium patients were identified according to the CAM. Sato et al. [7] evaluated pre- and post-operative risk factors for POD in consecutive 215 patients who received urological surgery. Inclusion criteria in this study were major and minor surgeries for benign and malignant diseases. Major surgery included radical prostatectomy, radical or partial nephrectomy, radical cystectomy, laparoscopic adrenalec- tomy, renal transplantation, ureterocystoneostomy, retroperitoneal sarcoma resection, and repair of urethra perineal fistula using a gracilis muscular flap after low anterior resection. Minor surgery included endoscopic transurethral resection of bladder tumor (TURBT), transurethral cystolithotomy, and high orchiectomy. Xue et al. [8] in their study evaluated 358 patients who underwent TURP under general or spinal anesthesia. All the studies were prospectively designed (table 1).

\section{Results}

\section{Incidence of Delirium}

The rate of the POD is variable depending on the type of surgical and anesthetic procedures [11]. The incidence of the POD is not consistent and fluctuates between 4.7 and $30 \%$ depending on the type of the urologic surgery $[7,8]$. When the incidence of the POD is estimated regardless of the type of the surgery (both open and endourological), the incidence is $4.7-8.8 \%$ [4, 7]. The main endourological operations that have been studied in conjunction with POD are TURP and TURBT, with the incidence of the POD $7.8-21.23 \%$ in TURP while $10 \%$ in TURBT patients [6-8]. The incidence in major open urologic operations is highest in nephrectomy and nephroureterectomy population $(30 \%)$, followed by partial nephrectomy $(10 \%)$, radical prostatectomy (10\%), while in radical cystectomy an incidence of 10-29\% has been reported $[5,7]$.

\section{Onset and Duration of Delirium}

All studies seem to agree that the onset of the POD often happens on the first postoperative day [4-8]. POD also occurs less frequently on the 2 nd postoperative day, or even on the 3 rd day (within 3 days) after the operation, while the most delayed POD is reported to occur between 5 to 7 days postoperatively $[5,7,8]$.

Concerning the duration of the POD, the study of Tognoni et al. [4] is in concordance with Tai et al. [6], with 
the symptoms lasting $3.0 \pm 0.8$ and $2.9 \pm 0.8$ days, respectively. In agreement to the studies above, Xue et al. [8] reported a slight longer duration of 1-4 days.

\section{Risk Factors}

Age Age had the most crucial role since it was apparent as a factor in every study. In the study of Tognoni et al. [4] patients with POD were significantly older (77 \pm 1.7 years old). Large et al. [5] found that the median age of patients developing delirium was 77.8 years [interquartile range (IQR) 73.5-83.5] versus 73.1 years (IQR 70.1-76.5) for those who did not develop delirium $(\mathrm{p}=0.02)$, with an overall incidence of $29 \%$. Multivariate analysis suggested only each additional year of age was associated with the occurrence of POD [odds ratio (OR) $1.52,95 \%$ confidence interval (CI) 1.04-2.22, p = 0.03]. The multivariate analysis of Tai et al. [6] also suggested that delirious patients were slightly, but significantly older ( 76.37 vs. 69.27 years, $\mathrm{p}<0.05)$. According to Sato et al. [7] age greater than 79 years ( $77-80$ years, $\mathrm{p}<0.001$ ) was significant in the occurrence of POD. Finally, in the study of Xue et al. [8], patients who developed POD on either of the postoperative days tended to be older (78.14 \pm 5.33 years) comparing to non-delirious (74.84 \pm 6.39 years).

CDT, IADL Assessment Tognoni et al. [4], as well as Tai et al. [6], showed that patients with POD had a poorer CDT score and were more impaired in the IADL assessment. Particularly in the study of Tognoni et al. [4], CDT score for delirium patients was $16.25 \pm 1.42$ compared to non-delirious patients $(18.37 \pm 0.29, \mathrm{p}=0.040)$. While in the study of Tai et al. [6], 16.01 versus $18.73(p=0.038)$ was found in the delirious patients when compared with the non-delirious group, respectively. IADL scores were in both studies much higher in the delirious patients when compared with the non-delirious group (6.54 vs. $3.01, \mathrm{p}=0.011$, in the study of Tai et al. [6] and $0.88 \pm$ 0.39 vs. $0.18 \pm 0.08, p=0.017$ in the study of Tognoni et al. [4], respectively).

Marital Status Tai et al. [6] also collected some other interesting data. In their study, the marital status had a significant role, since married percentage was significantly lower in the delirious patients when compared with the non-delirious group (51 vs. $86 \%, \mathrm{p}=0.013$ ).

Prior Hospitalization, GDS Tai et al. [6] showed that the prior hospitalization for other reason percent was much higher in the delirious patients compared with the non-delirious group (16.2 vs. 7.1\%, $\mathrm{p}=0.015)$. Furthermore, even though the preoperative ADL score in the de- lirious patients was similar to the non-delirious group, the GDS (and IADL as seen before) score was much higher in the delirious patients when compared with the non-delirious group (8.65 vs. 3.13, $\mathrm{p}=0.020$ for GDS score).

Preoperative MMSE Score Large et al. [5] were the only who found that preoperative MMSE score had a significant role in the development of POD and that the mean preoperative MMSE score for patients developing delirium was 24.6 (of a possible 30 ) versus 26.7 for those who did not $(\mathrm{p}=0.03)$.

Prostatitis-Like Symptoms (PLS), International Prostate Symptom Score (IPSS), and National Institutes of Health Chronic Prostatitis Symptom Index (NIH-CPSI) Tai et al. [6] also collected data for each patient, concerning IPSS and NIH-CPSI score. They found that IPSS and NIH-CPSI scores were both much higher in the delirious patients when compared with the non-delirious group (28.5 vs. $15.2, p=0.027 ; 37.8$ vs. $25.3, p=0.019$, respectively). Most significant in their study was the difference between the delirious and non-delirious group regarding the Nickel et al. [12] criteria. The significantly higher incidence of PLS was recorded in the delirious patients than in non-delirious ones (54.1 vs. $31.4 \%, \mathrm{p}=$ 0.009). According to the Nickel et al. [12], the patients are classified as having PLS with the NIH-CPSI if they complained of perineal and/or ejaculatory pain or discomfort, and their total index pain score is 4 or greater.

Handgrip Strength, Slower Gait Speed in the Timed Get-Up and Go Test and Fall Risk Assessment Frailty is considered to be a potential risk factor for delirium [7]. Patients suffering delirium had weaker handgrip strength $(\mathrm{p}=0.002)$, slower gait speed in the timed Get-up and Go test $(\mathrm{p}<0.001)$, and higher fall risk assessment ( $\mathrm{p}$ $=0.002$ ) than patients without delirium in preoperative factors. Gait speed slower than $13.0 \mathrm{~s}$, and fall risk score higher than 10 points were used for analysis in this study. No patients had experienced previous POD in this study. There was no difference between patients with or without delirium in gender, body mass index, and nutritional status between the groups.

$e G F R$ In postoperative factors, Sato et al. [7] also found that rapid eGFR decline $(\mathrm{p}=0.002)$ played a significant role in the occurrence of POD. Median eGFR change between preoperative and the day after surgery in radical prostatectomy, radical cystectomy, radical nephrectomy, partial nephrectomy, nephroureterectomy, other major surgeries (except for renal transplantation), and minor surgeries were $4.0,-9.0,-39,-21,-23,5$, and $1 \%$, respectively. 
Table 2. Review of risk factors associated with POD

\begin{tabular}{ll}
\hline Risk factors & $\begin{array}{l}\text { Reference } \\
\text { number }\end{array}$ \\
\hline Older age & {$[4-8]$} \\
Weaker handgrip strength & {$[7]$} \\
Slower gait speed in the timed Get-up and Go test & {$[7]$} \\
Higher fall risk assessment & {$[7]$} \\
Rapid eGFR decline & {$[7]$} \\
Higher VAS pain scores & {$[8]$} \\
Marital status (singles) & {$[6]$} \\
Prior hospitalization for other reason & {$[6]$} \\
Higher IPSS & {$[6]$} \\
Higher NIH-CPSI score & {$[6]$} \\
PLS based on the Nickel et al. criteria & {$[6]$} \\
GDS & {$[6]$} \\
IADL scores & {$[4,6]$} \\
Poorer CDT score & {$[4,6]$} \\
Preoperative MMSE score & {$[5]$} \\
Personal history of delirium & {$[4]$} \\
Significantly higher incidence of hypotension (intraoperatively) & {$[4]$}
\end{tabular}

Visual Analog Scale (VAS) Pain Scores According to the study of Xue et al. [8], patients who developed POD on either of the postoperative days had more comorbidity and experienced significantly higher VAS pain scores postoperatively, ranging from "no pain" $(0)$ to "very severe pain" (10). Pain intensity was measured at the same time that patients were evaluated for delirium (for postoperative day 1: mean postoperative VAS at rest $3.79 \pm$ 0.93 vs. $2.42 \pm 0.83, \mathrm{p}=0.021$; and for postoperative day 2: mean postoperative VAS at rest $2.71 \pm 0.82$ vs. $2.05 \pm$ $0.76, p=0.025)$. No differences were seen in delirious compared with non-delirious patients in anesthesia type in both groups.

Personal History of Delirium In the study of Tognoni et al. [4], the onset of transient delirium occurred on the first postoperative day and lasted $3.0 \pm 0.8$ days. The study also revealed that patients with POD had a greater personal history of delirium and, even though an intraoperative factor, there was a significantly higher incidence of hypotension recorded in delirious patients than in non -delirious patients $(5 / 8$ vs. 20/82; $\mathrm{p}<0.05)$ (table 2$)$.

\section{Discussion}

Delirium is associated with lower cognitive and functional levels during follow-up and increased lengths of hospital stay, costs, morbidity, and mortality rates [13,
14]. It is highly prevalent among the inpatient geriatric population but remains poorly studied in patients undergoing urologic surgery.

Previous reviews noted that the incidence of delirium ranges from 10 to $46 \%$ in general surgical population $[15,16]$. The risk factors included older age, cognitive impairment, illness severity, psychopathological symptoms, preoperative depression, preoperative psychotropic drug use, greater comorbidity, and perioperative complications [17].

Although many studies have described rates and risk factors for POD in major surgeries [18, 19], only a few reports have investigated rates and risk factors for POD in urological surgery.

In this review, we tried to identify the incidence as well as the potential risk factors which indicate the possibility of POD. However, all predictive factors cannot be applied to clinical practice without a well-trained geriatric care team, and therefore, an easy and simple method to ensure the quality of each assessment is required.

Huge methodological differences among studies are important drawbacks to diagnose delirium. In most of the studies [4-6], delirium was screened and diagnosed using the CAM, which may explain the higher incidence of delirium in comparison to the study of Sato et al. [7].

The incidence of POD after endoscopic urological surgery was associated with relative low rate of delirium, particularly $7.8 \%$ in the study of Xue et al. [8], which is similar to the study of Tognoni et al. [4] (incidence 8.8\%) that investigated 90 patients ( 81 males and 9 females; average age of $74.3 \pm 0.40$ years) undergoing urological surgery.

On the other side, the delirium incidence of $21.23 \%$ was found in patients of 60 years and older in the study of Tai et al. [6]. Still, these findings were lower than the study of Large et al. [5], who reported 29\% incidence in elderly patients after radical cystectomy and the study of Gani et al. [20] who reported a 26\% (166/640 patients) incidence in patients who underwent surgery at a urology clinic. The latter did not specify if the kind of operation had a key role in that.

Large et al. [5], where the only ones who statistically significant reported that, patients who developed delirium scored $>2$ points lower on preoperative MMSE testing than their non-delirious patients and had also a statistically larger decrease from baseline (about 3.5 points) in their MMSE scores than patients who did not develop delirium.

In the study of Sato et al. [7], risk factors identified were older age ( $>65$ years), impairment in the IADL, poor CDT, GDS, a previous history of delirium, and mental status examinations. 
Regarding the age, there are also neuro-chemical factors predis posing elderly patients for delirium, such as lack of cholinergic reserves [21]. This risk will be provoked by commonly used medications with anticholinergic activity such as ipratropi um and opiate analgesics.

Furthermore, the relationship between rapid renal function decline and POD leads to the hypothesis that the acute decline of renal function may induce accumulation of anesthetizing agents, opioids, and/or metabolic substances after unilateral nephrectomy [7]. Measuring the concentration of anesthetizing agent may help to stratify the risk for POD. Further study is required to address these issues. Another limitation is the difficulty to address the unmeasurable confounders including anesthetic procedures, dosages of anesthetic agents and dosages of opioids.

In the cohort study of Tai et al. [6], the CDT and GDS scores appear to be more sensitive in predicting POD than the MMSE score. The assessment of IADL was also more sensitive than ADL for the prediction of delirium, further suggesting that the evaluation of functional status is an important issue in the pre-surgery assessment [4, $22,23]$. However the above assessments, in contrast with the MMSE score assessment, might require a trained assistant to perform the measurements.

In the same study, consistent with other studies, patients with poor IPSS and NIH-CPSI scores and PLS are more prone to delirium after the TURP. Additionally, POD was associated with the single, widowed and divorced, as well as with the prior hospitalization for other reason, suggesting that the healthcare team should better include this factors in the preoperative assessment and that there are also risk factors cor related to psychological disorders prior to the operation and thus for developing POD.

Pain intensity after TURP was found to be a risk factor for delirium [8]. Data showed that patients with POD had significantly higher VAS scores for pain following TURP than those who were not delirious. The overall severity of postoperative bladder spasm can be managed with the use of opioids, anticholinergic medication, and sedatives. On the other hand, there is some controversy over whether opioid use may itself be a risk factor for delirium [24, 25]. Anticholinergic medications as well have long been recognized as a common risk factor for the development of delirium. By replacing opioids with other medications such as intravesical bupivacaine and by using tolterodine, which has a low incidence of central nervous system side effects, we can suppress postoperative bladder spasm without promoting POD [26].
All of the studies were in accordance with previous studies reported a low incidence of delirium after postoperative day 4 [27-29].

Tognoni et al. [4] found that in their group of patients POD occurred in $<10 \%$, confirming the hypothesis that urological surgery is associated with a low rate of delirium. The risk factors previously reported in the literature were confirmed in their study since older patients (77 vs. 74 years, $p<0.05$ ) and patients with initial cognitive impairment had a higher risk of developing POD. Moreover, the CDT appears to be more sensitive in predicting POD than MMSE, when an initial cognitive derangement is present.

Another risk factor associated with the POD in their study was the presence of a functional decline in IADL. IADL was also more sensitive than ADL for the prediction of delirium, further indicating that an initial functional deterioration is sufficient to contribute to the onset of delirium, as well as a previous history of delirium.

Tognoni et al. [4] revealed the finding from other studies that a previous history of delirium increases the probability of POD [30, 31].

Another statistically significant finding was that a significant intraoperative fall of blood pressure and hypotension contributes to the prediction of the POD, according to the findings of Marcantonio et al. [27]. However, this factor cannot be assessed before the surgical procedure and could only be addressed during the surgery, properly constituting an intraoperative risk factor.

Given their small sample size, these studies serve as hypothesis generating. Larger patient populations from multiple institutions will be able to characterize the incidence, treatment, and long-term consequences of POD in the urologic patients. Despite limitations, it is the first attempt for a review in this field, where a lack of knowledge exists, even though a high percentage of urological patients includes older people with cognitive issues.

Regarding methods and metrics, CAM is limited by its reliance on a simple observation performed once on a given day, creating a discontinuity in data collection because the patient is not under direct observation for long periods of time [32]. Furthermore, the CAM is just one tool for establishing the presence or absence of delirium, and it is unable to measure the severity or exact duration of delirium [33]. However, the CAM is very well validated against the diagnosis of delirium from the specialists [34].

The follow-up, using MMSE and CAM testing on postoperative care visits will help track cognitive decline or improvement in patients who become delirious and 
also to distinguish the role of delirium as potential morbidity or mortality factor.

In conclusion, the MMSE score and older age were significantly associated with the development of POD. Additionally, avoiding identified potential confounding factors as pain, anemia, infection, electrolyte disorders, hormonal disorder, neurological dysfunction, dehydration, renal failure, probably lowers the onset of POD. Combined, the above measures could help to identify delirious patients and reduce the cost, morbidity, and mortality in urologic patients after surgery.

\section{References}

1 Inouye SK: Delirium in older persons. $\mathrm{N}$ Engl J Med 2006;354:1157-1165.

2 Marcantonio ER, Kiely DK, Simon SE, John Orav E, Jones RN, Murphy KM, Bergmann MA: Outcomes of older people admitted to postacute facilities with delirium. J Am Geriatr Soc 2005;53:963-969.

3 Bucht G, Gustafson Y, Sandberg O: Epidemiology of delirium. Dement Geriatr Cogn Disord 1999;10:315-318.

4 Tognoni P, Simonato A, Robutti N, Pisani M, Cataldi A, Monacelli F, Carmignani G, Odetti P: Preoperative risk factors for postoperative delirium (POD) after urological surgery in the elderly. Arch Gerontol Geriatr 2011;52:e166-169.

5 Large MC, Reichard C, Williams JT, Chang C, Prasad S, Leung Y, DuBeau C, Bales GT, Steinberg GD: Incidence, risk factors, and complications of postoperative delirium in elderly patients undergoing radical cystectomy. Urology 2013;81:123-128.

6 Tai S, Xu L, Zhang L, Fan S, Liang C: Preoperative risk factors of postoperative delirium after transurethral prostatectomy for benign prostatic hyperplasia. Int J Clin Exp Med 2015;8:4569-4574.

7 Sato T, Hatakeyama S, Okamoto T, Yamamoto H, Hosogoe S, Tobisawa Y, Yoneyama T, Hashiba E, Yoneyama T, Hashimoto Y, Koie T, Hirota K, Ohyama C: Slow gait speed and rapid renal function decline are risk factors for postoperative delirium after urological surgery. PLoS One 2016;11:e0153961.

8 Xue P, Wu Z, Wang K, Tu C, Wang X: Incidence and risk factors of postoperative delirium in elderly patients undergoing transurethral resection of prostate: a prospective cohort study. Neuropsychiatr Dis Treat 2016;12:137-142.

9 Folstein MF, Folstein SE, McHugh PR: "Mini-mental state" A practical method for grading the cognitive state of patients for the clinician. J Psychiatr Res 1975;12:189-198.
10 American Psychiatric Association: Diagnostic and statistical manual of mental disorders: DSM-5, ed 5. Arlington VA, American Psychiatric Association, 2013, p947.

11 Papaioannou A, Fraidakis O, Michaloudis D, Balalis C, Askitopoulou H: The impact of the type of anaesthesia on cognitive status and delirium during the first postoperative days in elderly patients. Eur J Anaesthesiol 2005; 22:492-499.

12 Nickel JC, Downey J, Hunter D, Clark J: Prevalence of prostatitis-like symptoms in a population based study using the National Institutes of Health chronic prostatitis symptom index. J Urol 2001;165:842-845.

13 Marcantonio ER, Goldman L, Mangione CM, Ludwig LE, Muraca B, Haslauer CM, Donaldson MC, Whittemore AD, Sugarbaker DJ, Poss R: A clinical prediction rule for delirium after elective noncardiac surgery. JAMA 1994;271:134-139.

14 O'Keeffe S, Lavan J: The prognostic significance of delirium in older hospital patients. J Am Geriatr Soc 1997;45:174-178.

15 Mittal V, Muralee S, Williamson D, McEnerney N, Thomas J, Cash M, Tampi RR: Review: delirium in the elderly: a comprehensive review. Am J Alzheimers Dis Demen 2011;26:97-109.

16 Deiner S, Silverstein JH: Postoperative delirium and cognitive dysfunction. Br J Anaesth 2009;103(suppl 1):i41-46.

17 Dasgupta M, Dumbrell AC: Preoperative risk assessment for delirium after noncardiac surgery: a systematic review. J Am Geriatr Soc 2006;54:1578-1589.

18 Brouquet A, Cudennec T, Benoist S, Moulias S, Beauchet A, Penna C, Teillet L, Nordlinger B: Impaired mobility, ASA status and administration of tramadol are risk factors for postoperative delirium in patients aged 75 years or more after major abdominal surgery. Ann Surg 2010;251:759-765.
19 Smulter N, Lingehall HC, Gustafson Y, Olofsson B, Engstrom KG: Delirium after cardiac surgery: incidence and risk factors. Interact Cardiovasc Thorac Surg 2013;17:790-796.

20 Gani H, Domi R, Kodra N, Prifti P, Naco M, Beqiri V, Torba D, Tare R: The incidence of postoperative delirium in elderly patients after urologic surgery. Med Arch 2013;67:45-47.

21 Rudolph JL, Ramlawi B, Kuchel GA, McElhaney JE, Xie D, Sellke FW, Khabbaz K, Levkoff SE, Marcantonio ER: Chemokines are associated with delirium after cardiac surgery. J Gerontol A Biol Sci Med Sci 2008; 63:184-189.

22 Minden SL, Carbone LA, Barsky A, Borus JF, Fife A, Fricchione GL, Orav EJ: Predictors and outcomes of delirium. Gen Hosp Psychiatry 2005;27:209-214.

23 Korevaar JC, van Munster BC, de Rooij SE: Risk factors for delirium in acutely admitted elderly patients: a prospective cohort study. BMC Geriatr 2005;5:6.

24 Sieber FE, Mears S, Lee H, Gottschalk A: Postoperative opioid consumption and its relationship to cognitive function in older adults with hip fracture. J Am Geriatr Soc 2011;59:2256-2262.

25 Fong HK, Sands LP, Leung JM: The role of postoperative analgesia in delirium and $\operatorname{cog}$ nitive decline in elderly patients: a systematic review. Anesth Analg 2006;102:1255-1266.

26 Chiang D, Ben-Meir D, Pout K, Dewan PA: Management of post-operative bladder spasm. J Paediatr Child Health 2005;41:56-58.

27 Marcantonio ER, Goldman L, Orav EJ, Cook EF, Lee TH: The association of intraoperative factors with the development of postoperative delirium. Am J Med 1998;105:380-384.

28 Taub DA, Dunn RL, Miller DC, Wei JT, Hollenbeck BK: Discharge practice patterns following cystectomy for bladder cancer: evidence for the shifting of the burden of care. J Urol 2006;176:2612-2617. 
29 Amador LF, Goodwin JS: Postoperative delirium in the older patient. J Am Coll Surg 2005;200:767-773.

30 Inouye SK, Charpentier PA: Precipitating factors for delirium in hospitalized elderly persons. Predictive model and interrelationship with baseline vulnerability. JAMA 1996; 275:852-857.
31 Litaker D, Locala J, Franco K, Bronson DL, Tannous Z: Preoperative risk factors for postoperative delirium. Gen Hosp Psychiatry 2001;23:84-89.

32 Inouye SK, van Dyck CH, Alessi CA, Balkin S, Siegal AP, Horwitz RI: Clarifying confusion: the confusion assessment method. A new method for detection of delirium. Ann Intern Med 1990;113:941-948.
33 Monette J, Galbaud du Fort G, Fung SH, Massoud F, Moride Y, Arsenault L, Afilalo M: Evaluation of the Confusion Assessment Method (CAM) as a screening tool for delirium in the emergency room. Gen Hosp Psychiatry 2001;23:20-25.

34 Inouye SK, Bogardus ST Jr, Charpentier PA, Leo-Summers L, Acampora D, Holford TR, Cooney LM Jr: A multicomponent intervention to prevent delirium in hospitalized older patients. N Engl J Med 1999;340:669-676. 